\title{
The Effects of Evening Bright Light Exposure on Subsequent Morning Exercise Performance
}

Authors

Affiliations

\author{
A. Thompson ${ }^{1,2}$, H. Jones ${ }^{2}$, E. Marqueze ${ }^{3}$, W. Gregson ${ }^{2}$, G. Atkinson ${ }^{4}$
}

Affiliation addresses are listed at the end of the article

\author{
Key words \\ circadian rhythms \\ - thermoregulation \\ - melatonin \\ - performance \\ pre-cooling
}

accepted after revision

August 01, 2014

Bibliography

DOI http://dx.doi.org/

10.1055/s-0034-1389970

Published online:

October 6, 2014

Int J Sports Med 2015; 36:

101-106 @ Georg Thieme

Verlag KG Stuttgart · New York

ISSN 0172-4622

\section{Correspondence}

\section{Dr. Andrew Thompson}

Molecular and Clinical

Pharmacology

University of Liverpool

Block A Waterhouse Building

Liverpool

United Kingdom

L69 3GL

Tel.: +44/151/795 5390

Fax: +44/151/795 5390

andrew.thompson@liverpool. ac.uk

\section{Abstract \\ $\nabla$}

We investigated the effects of evening bright light on the circadian timing of core temperature and morning exercise performance under conditions of high thermal stress. At 20:00 h, 8 males were exposed to a standardised light protocol and thereafter to either polychromatic bright light (2500 lux at $50 \mathrm{~cm}, \mathrm{BL})$ or no light (0 lux, NL) for $30 \mathrm{~min}$. The following morning, intermittent cycling exercise was undertaken followed by a $10 \mathrm{~km}$ time-trial in an environmental chamber set to $35^{\circ} \mathrm{C}$ and $60 \%$ relative humidity. Core body temperature was measured throughout. Data

\section{Introduction \\ $\nabla$}

It is widely accepted that exposure to ocular bright light is the most important zeitgeber for circadian entrainment in humans $[11,15]$. Depending on relative 'body clock time' at the time of administration, exposure to bright light can result in phase delays (to later times) or advances (to earlier times) of circadian rhythmicity in many physiological functions. For example, exposure to light in the hours before the nadir of the daily rhythm in core body temperature induces a phase delay, whereas light after the nadir advances the rhythm [15]. These responses can be described across a full 24-h cycle to provide a phase response curve to light, which is now well described [25].

In sport and exercise, it is common for long-distance running and cycling events to be scheduled in the morning, one reason being that athletes might be less prone to thermal stress. This scheduling is based, in part, on the knowledge that during competition under high ambient temperatures, a primary objective is to keep core body temperature low, given the high correlation between the onset of fatigue and reaching a 'critical' core temperature [16]. Pre-cooling interven- were analysed using a within-subjects model and presented as mean \pm SD. Time of the sleep-trough in core temperature occurred $\sim 1.75 \mathrm{~h}$ later following $\mathrm{BL}(\mathrm{P}=0.07)$. Prior to time-trial, core temperature was $0.27 \pm 0.42{ }^{\circ} \mathrm{C}$ lower in $\mathrm{BL}(95 \% \mathrm{CI}$ : -0.02 to $0.57, \mathrm{P}=0.07)$. The time-trial was completed $1.43 \pm 0.63 \mathrm{~min}(0.98-1.87)$ faster in $\mathrm{BL}(\mathrm{P}=0.001)$. Post time-trial, intestinal temperature was $38.21 \pm 0.56^{\circ} \mathrm{C}(37.84-38.57)$ in $\mathrm{BL}$ compared to $38.64 \pm 0.42^{\circ} \mathrm{C}(38.34-38.93)$ in $\mathrm{NL}(\mathrm{P}=0.10)$. These data provide the first evidence that a 30-min exposure to bright light prior to sleep can influence exercise performance under hot conditions during the subsequent early morning.

tions have been shown to be effective in reducing thermal stress in athletes, alongside mediating lower cardiovascular and psychophysical strain $[7,13,24]$. Furthermore, the naturally lower core body temperature in the morning hours has been postulated as a reason for improved performance under high ambient temperatures. Such results are paradoxical to many instances of sport/exerciserelated performance, which often peaks in the evening [30]. In support of the notion that "evening is not always best”, Hobson, et al. [20] reported a greater time to exhaustion under high temperatures by around $5 \mathrm{~min}$ at $06: 45 \mathrm{~h}(45.8 \pm 10.7 \mathrm{~min})$ compared to $18: 45 \mathrm{~h}(40.5 \pm 9.0 \mathrm{~min})$, with initial core body temperature being $\sim 0.1^{\circ} \mathrm{C}$ lower in the morning.

In a recent study, Atkinson, et al. [1] hypothesised that carefully planned bright light exposure could manipulate circadian time and function to 'pre-cool' an athlete prior to endurance exercise under hot conditions. They reported that evening bright light delayed the time that core body temperature fell to its minimum during sleep, leading to a lower core temperature prior to exercise the following morning. Nevertheless, this study could not confirm whether the changes in core body temperature actually translated to 
improved performance as no performance-related outcome was measured.

The relationships between bright light, circadian rhythmicity and endurance performance in the heat are still enigmatic. Therefore, the aim of the present study was to manipulate core body temperature by exposing participants to bright light with short wavelength characteristics prior to the nocturnal sleep period. We hypothesised that evening exposure to bright light results in (i) melatonin suppression, (ii) a delay in the circadian timing of core body temperature, consequently leading to a lower internal temperature immediately prior to and during exercise undertaken on the subsequent morning, and (iii) improved endurance performance due to this 'pre-cooling' intervention.

\section{Methods}

$\nabla$

\section{Participants}

8 males were recruited for the present study. The mean \pm SD age, body mass, height and maximal oxygen uptake $\left(\dot{\mathrm{VO}}_{2 \max }\right)$ of the participants was $22 \pm 2$ years, $80.8 \pm 10.37 \mathrm{~kg}, 1.82 \pm 0.10 \mathrm{~m}$ and $44.9 \pm 2.5 \mathrm{ml} \cdot \mathrm{kg}^{-1} \cdot \mathrm{min}^{-1}$, respectively. All participants were healthy, non-smokers and none reported having a history of major illness or sleeping problems. All were involved in regular recreational level sport/exercise activities; none were training for or competing in events that included cycling. No participant was involved in nocturnal shift work and none had undertaken transmeridian travel during the 30 days prior to the study commencing. Participants were asked to refrain from alcohol, caffeine, coco-based products, bananas and strenuous exercise $24 \mathrm{~h}$ prior to attending the laboratory for each trial. Conventional workday nychthemeral wake-up and retiring to bed times were reported as 06:45-08:30 h and 22:45-00:15 h respectively. Participants were asked to maintain their regular weekday sleepwake cycle $48 \mathrm{~h}$ prior to testing. Written informed consent was obtained, and all procedures were approved by the ethics committee at Liverpool John Moores University and adhered to the declaration of Helsinki and the ethical guidelines of IJSM [19].

\section{Laboratory protocols}

Participants visited the laboratory on 3 separate occasions, the first for familiarisation purposes and measurement of maximal oxygen consumption, followed by 2 further visits to complete the main experimental conditions. Data collection for this study took place during the winter and early spring months in the UK, November-April.

During the initial visit, participants were familiarized with our sleep laboratory and all measurement tools and apparatus to be used during the experimental procedures. Anthropometric measures and resting blood pressure (mercury sphygmomanometer) were recorded during this visit. Resting blood pressure was measured with the participant seated following $5 \mathrm{~min}$ of quiet rest. All participants were normotensive. A maximal oxygen consumption test was completed on a cycle ergometer (Premier 8i Ergo_bike, Daum Electronics, Fürth, Germany) using a progressive continuous protocol (Bird \& Davidson, 1997). Power output was set initially at $75 \mathrm{~W}$ and increased by $25 \mathrm{~W}$ every 2 min until volitional exhaustion was reached or the required cadence could not be maintained ( $\left.\geq 60 \mathrm{rev} \cdot \mathrm{min}^{-1}\right)$. Oxygen consumption was measured on a breath-by-breath basis using an online gas analyser (Oxycon, Pro, Jaeger, Germany). Heart rate (RS400, Polar, Finland) and ratings of perceived exertion (RPE)
[8] were recorded at the end of each stage. The workload for the subsequent trials was calculated as $55 \% \mathrm{VO}_{2 \max }$ that was achieved in this test.

\section{Experimental trials}

Trial ordered was counterbalanced and separated by 5-11 days. Participants arrived at the laboratory at 19:30 h and adopted a semi-supine seated position, which was maintained for the entire evening testing period, this allowed for measures to return to baseline prior to the light protocol commencing. Following instrumentation, lighting in the laboratory was reduced to $<12$ lux for $45 \mathrm{~min}$ at 20:00 h. This period was followed by $1 \mathrm{~h}$ of no light. The laboratory lighting was switched off, and a commercially available eye-mask was worn to ensure no residual light was able to reach the retina. This period was followed by $30 \mathrm{~min}$ of intervention. Participants were exposed to one of two conditions during each visit: 1) the no-light condition continued (no light condition, NL). 2) exposure to 2500 lux of polychromatic light from a light box (Zip, Lumie, Cambridge, UK). The light box was placed $50 \mathrm{~cm}$ from the nasal root, and participants were instructed not to look directly into the light box for the whole intervention period but instead to gaze at the light for short intermittent periods and to keep the light in their periphery for the remainder of the time (bright light, BL). Following the intervention period, participants were administered dim light for a further $75 \mathrm{~min}$ before retiring to bed at 23:45 h. Participants slept in the laboratory in shorts and t-shirt or vest with a 10.5 tog duvet. Prior to sleep, participants were allowed to use the toilet and brush their teeth.

At $06: 30 \mathrm{~h}$ on the following morning, the participants were woken by a researcher and provided a saliva sample. Participants then got out of bed and drank $568 \mathrm{ml}$ of water and ingested a standardised snack. To recover from any sleep inertia, participants then rested for $30 \mathrm{~min}$ [32] before moving to an environmental chamber, which was pre-set to a temperature of $35^{\circ} \mathrm{C}$ and $60 \%$ humidity. Thermistors (120046, ELAB A/S, Copenhagen, Denmark) were attached to the participant. A 2-min baseline recording period was then conducted prior to exercise. The exercise protocol was intermittent and comprised three 10-min bouts of upright cycling at $55 \% \mathrm{VO}_{2}$ max on a cycle ergometer. Each bout was interspaced with $10 \mathrm{~min}$ of seated passive rest. Two min following each exercise bout a saliva sample was collected. Water was then available for consumption until $5 \mathrm{~min}$ into the rest period. Following the completion of the intermittent protocol, participants undertook a $10 \mathrm{~km}$ self-paced timetrial. During this time-trial, water was available ad libitum and the resistance on the cycle ergometer was self-selected. Throughout exercise and the time-trial, ratings of perceived exertion (RPE) were recorded using the Borg scale [8].

\section{Measurement procedures}

At $15: 00 \mathrm{~h}$ ( $5 \mathrm{~h}$ prior to attending the laboratory), participants were issued a silicon-coated thermometric pill (CorTemp, Human Technologies International, USA) and instructed to swallow it with $50 \mathrm{ml}$ of water. Each pill contained a crystal quartz oscillator which transmitted a low-frequency radio wave to an external data logger (HT150001, CorTemp, Human Technologies International, USA) attached to the participants' waist. The thermometric pill was used to record Intestinal temperature $\left(T_{c}\right)$. The pill was given prior to the participant visiting the laboratory to ensure that it had travelled through the body to the intestines and a digital reading was being received. 
Saliva samples were collected at numerous stages during the lighting protocol, after waking and during exercise. The samples were immediately frozen $\left(-80^{\circ} \mathrm{C}\right)$ and later analysed for melatonin concentration from duplicate samples using an enzymelinked immunosorbent assay kit (Direct saliva melatonin ELISA, Buhlmann, Schonenbuch, Switzerland). The correlation of saliva melatonin levels to other samples (blood plasma and urine) has been determined as acceptable [6].

Skin thermistors were attached to the participants' upper chest, mid forearm, upper thigh and medial side of the calf using sweat-proof tape (Transpore, 3M, Loughborough, England). A data logger (TM9616, ELLAB, Copenhagen, Denmark) recorded the temperature of each skin thermistor. Weighted mean skin temperature $\left(\mathrm{T}_{\mathrm{sk}}\right)$ was later calculated [29].

\section{Statistical analysis}

Our primary outcome was the time to complete the $10 \mathrm{~km}$ timetrial. Such protocols have been found to be highly reliable with test-retest coefficients of variation $(\mathrm{CV})<2 \%$ [3]. Using the NQuery software (Cork, Ireland), it was estimated that 8 subjects would facilitate the detection of a statistically significant difference between trials of $3.3 \%$ assuming a CV of $2 \%, P<0.05$ and $80 \%$ power with a two-tailed paired t-test.

Data collected prior to the evening light intervention phase were averaged and entered into the hypothesis test as a single covariate (Altman, 1991). Intestinal temperature collected post-intervention and during sleep was binned into $15 \mathrm{~min}$ blocks. Post-waking exercise and time-trial temperature data $\left(T_{c}\right.$ and $\mathrm{T}_{\mathrm{sk}}$ ) was binned depending on phase/stage (i.e., each $10 \mathrm{~min}$ exercise and rest period and each $2 \mathrm{~km}$ travelled, respectively). Data were analysed using two-factor within-subjects (trial $\times$ time) generalized estimation equations [4], with order of trial effect controlled within the model. The statistical outcome from the time-trial was used alongside the sample size information above to provide a magnitude-based inference for our primary variable [5]. One important summary statistic was an estimate of the time of minimum $\mathrm{T}_{\mathrm{c}}$ during sleep. Intestinal core temperature data were averaged into 15 -min time periods for data smoothing purposes. We recorded the absolute value and the time of the lowest of these 15-min averages as an estimate of the core body temperature minimum during sleep. The data were analysed using Statistical Package for Social Sciences (SPSS) for Windows (Version 17, SPSS Inc., Chicago, IL, USA). Data are presented as mean $\pm \mathrm{SD}(95 \% \mathrm{CI})$. The alpha level of significance was set at $P \leq 0.05$.

\section{Results}

\section{$\nabla$}

\section{Light intervention and sleep}

Exposure to bright light in the early biological night prior to the exercise day suppressed the rise in salivary melatonin concentration ( $\bullet$ Fig. 1). However, the difference between conditions did not reach statistical significance $\left[-9.0 \pm 25.4 \mathrm{pg} \cdot \mathrm{ml}^{-1}\right.$ $(-29.3-11.3, P=0.35)]$. Immediately after waking at $06: 30 \mathrm{~h}$, the differences in melatonin concentrations were negligible $\left[\mathrm{BL}=23.2 \pm 10.0 \mathrm{pg} \cdot \mathrm{ml}^{-1}(16.3-30.1)\right.$ vs. $\mathrm{NL}=24.9 \pm 5.9 \mathrm{pg} \cdot \mathrm{ml}^{-1}$ (20.8-29.0), $P=0.54]$.

The absolute minima value at the core temperature nadir did not significantly differ between conditions $\left[\mathrm{L}=36.36 \pm 0.21^{\circ} \mathrm{C}\right.$ (36.21-36.50) vs. $\left.\mathrm{NL}=36.36 \pm 0.35^{\circ} \mathrm{C}(36.12-36.61), P=0.95\right]$. However, the different timing of this phenomenon between con-

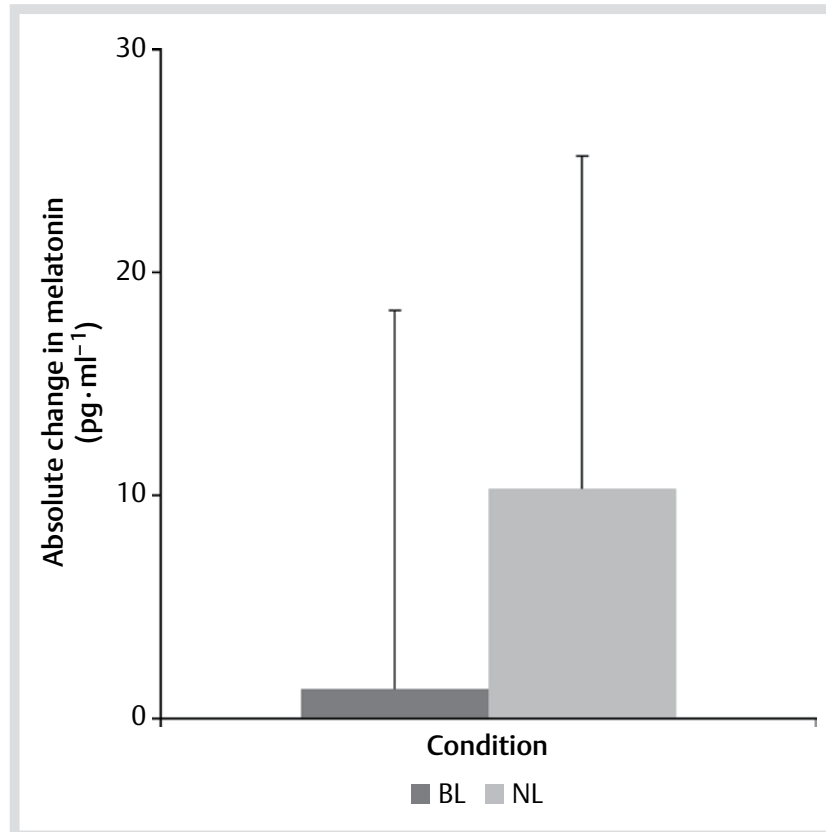

Fig. 1 Mean \pm SD salivary melatonin concentration changes in response to evening bright light or no light condition.

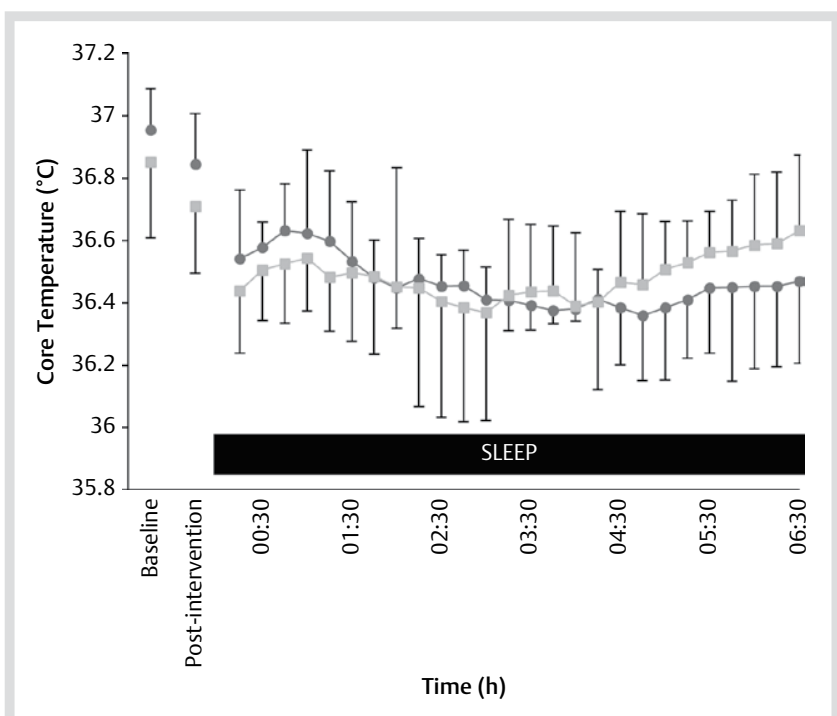

Fig. 2 Mean $\pm S D T_{c}$ measured at rest, post light intervention and during sleep. -•- Bright light condition - - - No light condition.

ditions was of practical relevance, occurring at approximately $04: 30 \mathrm{~h}$ in $\mathrm{BL}$ compared with $02: 45 \mathrm{~h}$ in NL $(P=0.07)$ ( $\odot$ Fig. 2). This resulted in a $0.16 \pm 0.30^{\circ} \mathrm{C}(-0.37-0.05, P=0.13)$ lower $\mathrm{T}_{\mathrm{C}}$ in BL compared with NL immediately prior to waking.

\section{Exercise and time-trial}

During the intermittent exercise $\mathrm{T}_{\mathrm{c}}$ increased. In the BL condition $\mathrm{T}_{\mathrm{c}}$ was $0.27 \pm 0.42^{\circ} \mathrm{C}(-0.57-0.02, P=0.06)$ lower than NL immediately prior to the initiation of the $10 \mathrm{~km}$ time-trial. There was evidence that the differences observed in $\mathrm{T}_{\mathrm{c}}$ between conditions increased as the time-trial progressed $(P<0.0005)(\odot \mathrm{Fig} .3)$, with a significant difference between conditions $(P=0.047)$. At the end of the time-trial intestinal temperature was $38.21 \pm 0.56^{\circ} \mathrm{C}$ (37.84-38.57) in BL compared with $38.64 \pm 0.42^{\circ} \mathrm{C}$ (38.3438.93), $P=0.10$. Changes in mean $T_{\text {sk }}$ were similar to those 


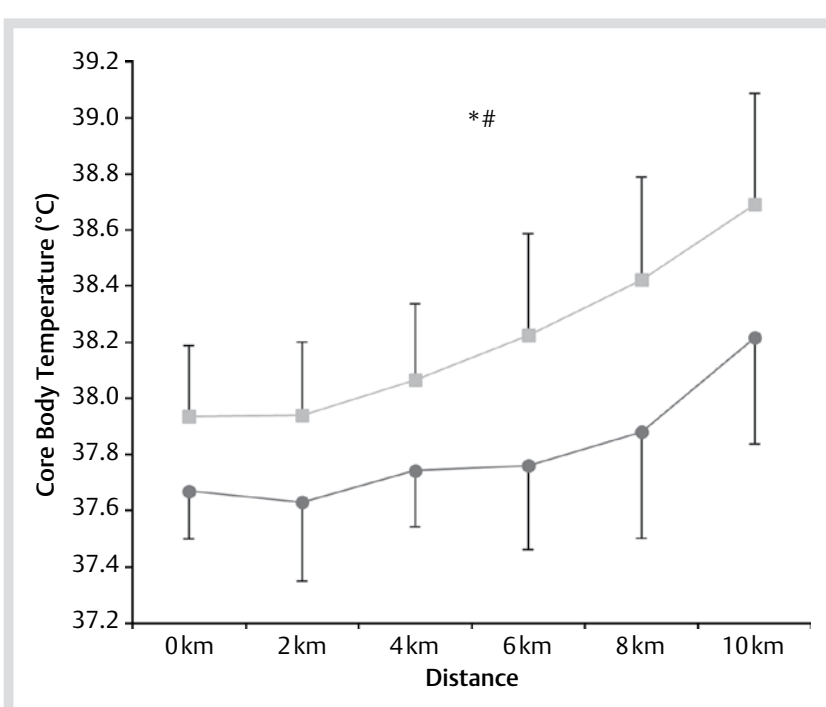

Fig. 3 Mean $\pm S D T_{c}$ measured prior to and during $10 \mathrm{~km}$ time-trial. * denotes significant effect of condition. \# denotes significant effect of time.

-•- Bright light condition - - - No light condition.

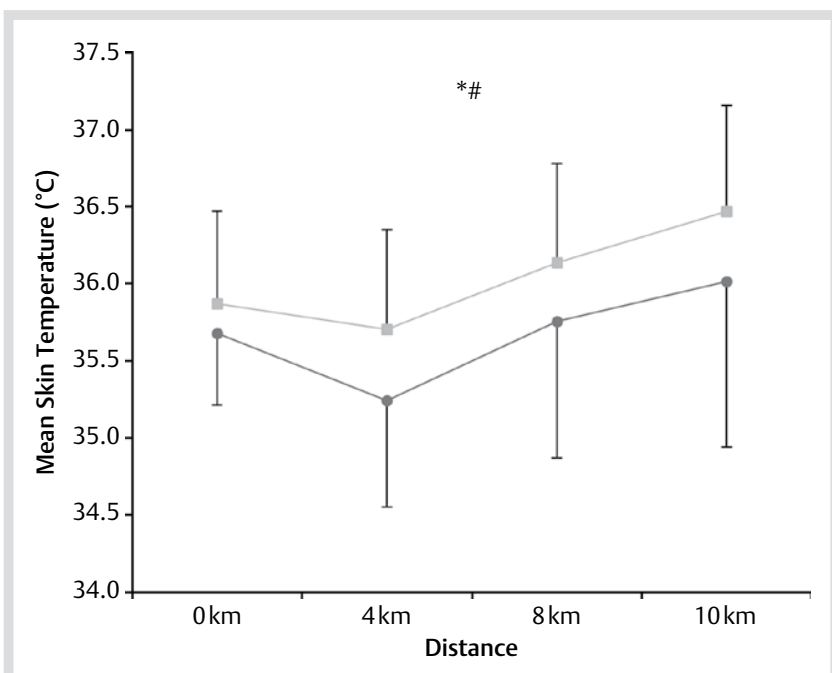

Fig. 4 Mean $\pm S D$ mean $T_{\text {sk }}$ measured prior to and during $10 \mathrm{~km}$ timetrial. * denotes significant effect of condition. \# denotes significant effect of time. - - Bright light condition - - No light condition.

observed in $\mathrm{T}_{c}$, with a highly significant increase over time $(P<0.0005)$. There was a significant overall difference between groups, with $\mathrm{BL} 0.26 \pm 0.33^{\circ} \mathrm{C}(0.03-0.49, P=0.03)$ lower on average ( $\bullet$ Fig. 4 ).

At the $2 \mathrm{~km}$ stage of the time-trial participants reported statistically significant lower ratings of perceived exertion in NL compared with $\mathrm{BL}[\mathrm{BL}=15.00 \pm 2.03(13.59-16.41) \mathrm{vs} . \mathrm{NL}=13.85 \pm 2.15$ (12.37-15.34), $P=0.02$ ]. Throughout the rest of the time-trial the differences in RPE were negligible between the two conditions (० Fig. 5).

Participants completed the $10 \mathrm{~km}$ time-trial $1.43 \pm 0.63 \mathrm{~min}$ (0.98-1.87, $P=0.001)$ faster in the bright light condition. This trend was observed throughout the time-trial, while at the $2 \mathrm{~km}$ stage the difference did not reach statistical significance $(P=0.15)$ (॰ Table 1).

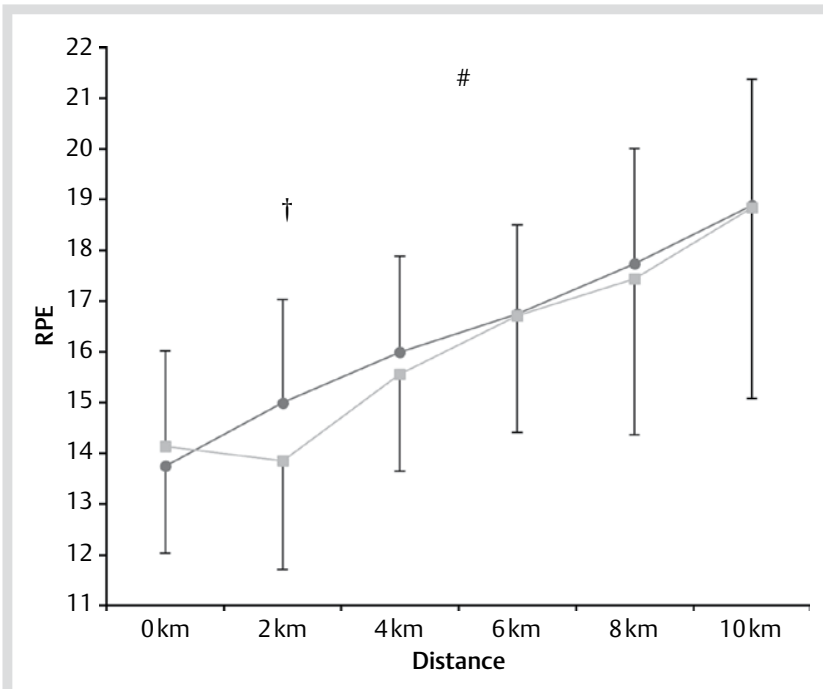

Fig. 5 Mean \pm SD RPE measured prior to and during $10 \mathrm{~km}$ time-trial. $\dagger$ denotes significant difference between conditions at time point. \# denotes significant effect of time. - •- Bright light condition - - - No light condition.

\section{Discussion}

$\nabla$

The novel finding in the present study is that $30 \mathrm{~min}$ of polychromatic light at an intensity of 2500 lux, $1 \mathrm{~h}$ prior to nocturnal sleep, mediated changes in $\mathrm{T}_{\mathrm{c}}$ and improved cycling performance in a $10 \mathrm{~km}$ time-trial the following morning. These data exhibit the importance of a lower core body temperature before and during exercise in the heat and how light can be used to facilitate adjustments in the homeostatic set-point of core body temperature. Such findings have implications for athletes competing in early morning events under conditions of high thermal stress. Our group has shown that exogenous melatonin lowers $\mathrm{T}_{\mathrm{c}}$ during subsequent intermittent exercise [2]. In more recent work, evening bright light led to a lower $\mathrm{T}_{\mathrm{c}}$ on the subsequent morning during $40 \mathrm{~min}$ of exercise in the heat [1]. The current study was the first to observe the effects of a bright light protocol on cycling performance under high ambient temperatures immediately after waking. To the authors' knowledge, this is also the first study to examine the residual effects of evening bright light on subsequent early morning physical performance.

The exposure to ocular bright light in the early biological night transiently attenuated the "normal" decrease in $\mathrm{T}_{\mathrm{c}}$. At the same time, the "normal" rise in melatonin, associated with healthy individuals [9], was suppressed. It is important to note, however, that light may exert it effects independently from those of melatonin. In agreement with other researchers [12,23], the transient increase in $T_{c}$ found in the bright light condition led to a delayed timing in the $T_{c}$ minimum. Previous studies observing the effects of bright light on the timing of the core temperature nadir have reported results of different magnitudes. For example, delays in the core temperature rhythm of $1.12 \mathrm{~h}, 0.75 \mathrm{~h}$ and $1.46 \mathrm{~h}$ following exposure to ocular bright light in late-evening/ early-night were observed by Kubota et al. [23], Krauchi et al. [22] and Atkinson et al. [1], respectively.

The inherent differences in study design may partly explain the aforementioned discrepancies. The timing ("real world" clock vs. body clock), intensity, duration and spectral distribution of light alongside prior light-dark exposure have all been hypothesised 


\begin{tabular}{|llllll|}
\hline & $\mathbf{2} \mathbf{k m}$ & $\mathbf{4 k m}$ & $\mathbf{6 k m}$ & $\mathbf{8} \mathbf{k m}$ & $\mathbf{1 0} \mathbf{~ k m}$ \\
\hline trial & & & & & \\
\hline light & $04.02 \pm 00.96$ & $07.93 \pm 01.60$ & $11.73 \pm 02.02$ & $15.15 \pm 01.90$ & $18.67 \pm 02.15$ \\
\hline no light & $04.26 \pm 00.60$ & $08.47 \pm 01.42$ & $12.67 \pm 02.20$ & $16.37 \pm 02.20$ & $20.10 \pm 02.48$ \\
\hline$P$-value & 0.15 & $<0.0005$ & $<0.0005$ & $<0.0005$ & $<0.0005$ \\
\hline
\end{tabular}

1 Cumulative time taken to complete each $2 \mathrm{~km}$ stage of the $10 \mathrm{~km}$ time-trial (minutes:seconds). Data presented as mean \pm SD. as contributing factors which influence the magnitude of phaseshifting [15]. In addition, sampling error may affect results, especially with a small sample size. In our study, the bright light condition caused a delay of approximately $1.75 \mathrm{~h}$ in the temperature minimum, which resulted in a $0.16^{\circ} \mathrm{C}$ lower $\mathrm{T}_{\mathrm{c}}$ during the final $15 \mathrm{~min}$ of nocturnal sleep. It should be noted that in studies which involve attempted circadian manipulation and exercise, directly measuring the effects of the interventional product on any changes in the full circadian rhythm of core body temperature is not feasible. This is due to the inherent study aim of undertaking exercise. It is well documented that physical activity produces masking effects on circadian rhythms, making estimates of circadian phase unreliable.

Following a $30 \mathrm{~min}$ period to reduce the effects of transient sleep inertia [32] and three $10 \mathrm{~min}$ bouts of light-moderate exercise, the difference in $\mathrm{T}_{\mathrm{c}}$ increased to $\sim 0.3^{\circ} \mathrm{C}$. The lower starting $\mathrm{T}_{\mathrm{c}}$ in the bright light condition resulted in significantly faster time to completion in the time-trial. A lower temperature at the start of exercise has been shown to improve cycling performance in conditions of high thermal stress. Hobson et al. [20] postulated that a lower rectal temperature at $06: 45 \mathrm{~h}$ compared to $18: 45 \mathrm{~h}$ resulted in participants being able to exercise 5 min longer until reaching exhaustion. Atkinson et al. [1] manipulated a lower core body temperature prior to exercise through bright light, which resulted in decreased rates of perceived exertion.

High levels of hyperthermia have been associated with the onset of fatigue, which was indexed in the present study by high RPE scores. Gonzalez-Alonso et al. [16] reported that lower starting core temperature resulted in a longer time to exhaustion in the heat, although a critical temperature of just above $40^{\circ} \mathrm{C}$ was observed across all trials. It was postulated that a higher core temperature resulted in a decline in cardiac output, attributed to a larger reduction in stroke volume via increased skin blood flow and skin blood volume. However, other factors have been hypothesised in the aetiology of hyperthermia-induced fatigue. These factors include, among others, deviations from the homeostatic set-point in brain temperature [27], hydration status, metabolic regulation and/or central nervous system control [18].

Pre-cooling is adopted by many athletes as a method for improving performance. In a recent meta-analysis, which included 27 studies, Wegmann et al. [33] concluded that firstly, precooling is more effective at improving performance in hot $\left(>26^{\circ} \mathrm{C}\right.$; $\left.+6.6 \%\right)$ compared to moderate temperatures (18$26^{\circ} \mathrm{C},+1.4 \%$ ); Secondly, pre-cooling prior to time-trials improves performance, on average, by $+4.2 \%$; Thirdly, the methods utilised (and changes in performance) in the selected studies were cold drinks $(+15.0 \%)$, cooling packs $(+5.6 \%)$, a cooled room $(+10.7 \%)$, cooling vests $(+4.8 \%)$ and water application $(+1.2 \%)$. In the present study a $7.2 \%$ improvement was observed over a $10 \mathrm{~km}$ cycling time trial. This effect can be considered large and to be the result of a modest reduction in core body temperature. In the studies included in this meta-analysis, there was not a linear relationship between changes in body temperature and performance. For example, a reduction in rectal temperature of $0.2^{\circ} \mathrm{C}$ produced an improvement in running performance of $1.1 \%$ (Arngrimsson et al., 2004), and a reduction of $0.51{ }^{\circ} \mathrm{C}$ in core temperature improved cycling performance by $6.5 \%$ (Ihsan et al., 2010). Conversely, a negligible change in core temperature and a $10.1 \%$ improvement in performance were observed by Duffield et al. (2003). The type of performance test also needs to be considered when interpreting results [3]. However, time-trials often have smaller percentage changes than time to exhaustion tests. The confidence interval for difference in time at the end of the time-trial highlights that the population mean difference could be as small as 0.98 and as large as $1.87 \mathrm{~min}$. It is likely that even the lower limit of this confidence interval is practically significant at this duration. This is confirmed by the use of magnitude-based inference on our primary outcome (time to complete the $10 \mathrm{~km}$ time-trial), which indicated that the chance of our difference being practically important was "very likely" (98.9\%).

Mean skin temperature generally followed a similar profile as core body temperature, with temperatures increasing as the time-trial progressed and higher temperatures being achieved in NL. Given the association with increased core temperature and the up-regulation of heat loss mechanisms via the skin, this finding is to be expected. High skin temperature has been proposed as an important rate-limiting factor in exercise intensity $[21,31]$. Whether core or skin temperature is a better marker of exercise capacity is yet to be fully substantiated.

Ratings of perceived exertion increased over time, although there were no discernible differences between conditions at the end of the time-trial. This finding is not surprising as individuals were asked to performance close to maximal capacity under very high ambient temperatures to which they were not accustomed. Therefore, the lower body temperature noted in BL may have reduced comparative thermal strain leading to increased effort, proportionately improving time to completion during the time-trial. Indeed, Schlader et al. [31] highlighted that a reduction in exercise intensity may occur as a safeguard against higher perceived exertion in the heat.

Beside Atkinson et al. (2008), only one other study has observed the effects of bright light on responses to exercise. O'Brien and O'Connor [28] concluded that physiological and subjective responses to $20 \mathrm{~min}$ of stationary cycling were unaffected by light. However, direct comparisons between this and the current study are difficult as the light conditions were altered acutely during exercise rather than for circadian manipulation. Furthermore, no information was provided about the ambient conditions in which exercise was performed.

Research utilising bright light as an intervention is not without pitfalls. For example, it is difficult to blind participants to conditions. In the present study it could be argued that a treatment effect was observed due to there being no true placebo, but the participants were not informed of the experimental rationale. In addition, the researchers conducting the exercise test were not blinded to the previous lighting condition. These researchers were instructed to give minimal motivational feedback to the participant to help reduce any potential bias. 
Interestingly, immediately after waking there were minimal differences in the observed salivary melatonin concentrations between conditions. This is surprising given the altered rate of melatonin suppression following light exposure the previous evening and the substantiated inverse relationship between core body temperature and melatonin [10]. One possible factor which may have influenced the lack of variation between groups postwaking is postural change. When the samples were drawn upon waking, some participants remained in bed for a short time, while others rose immediately. Postural condition may well have varied within subjects, influencing melatonin concentrations. The effects of posture on melatonin have been reported by previous studies $[14,26]$. Deacon and Arendt (1994) were the first to observe such effects and found that levels rise with standing after being supine and fall when the reverse posture is adopted. The mechanism behind this phenomenon, especially for salivary melatonin, remains unclear. However, it has been postulated that the gravitational forces associated with postural changes (e.g. supine to standing) may influence the human circulatory system [17] and in turn hormone concentrations.

In summary, the results presented in this study demonstrate the potential utility of bright light exposure prior to sleep as an ergogenic aid for endurance performance under high ambient temperatures during morning training and/or competition. A lower $\mathrm{T}_{\mathrm{c}}$ prior to waking was manipulated, which led to improved $10 \mathrm{~km}$ cycling time-trial. This technique has the potential to be more comfortable for the individual than other, more traditional, 'pre-cooling' techniques which often involve cold-packs on the skin or partial/full immersion in ice-baths. Further experimental data from elite athletes is required to fully understand the potential benefits of this interventional product.

\section{Acknowledgements \\ $\nabla$}

We are grateful to Lumie for providing financial support for this study. The authors have no financial interest associated with the outcome of this study and declare that they have no conflict of interest.

\section{Affiliations}

Molecular and Clinical Pharmacology, University of Liverpool, Liverpool, United Kingdom

Research Institute for Sport and Exercise Sciences, Liverpool John Moores University, Liverpool, United Kingdom

School of Public Health, University of São Paulo, São Paulo, Brazil

Health and Social Care Institute, Teesside University, Middlesbrough, United Kingdom

\section{References}

1 Atkinson G, Barr D, Chester N, Drust B, Gregson W, Reilly T, Waterhouse $J$. Bright light and thermoregulatory responses to exercise. Int J Sports Med 2008; 29: 188-193

2 Atkinson G, Holder A, Robertson C, Gant N, Drust B, Reilly T, Waterhouse $J$. Effects of melatonin on the thermoregulatory responses to intermittent exercise. J Pineal Res 2005; 39: 353-359

3 Atkinson G, Nevill AM. Selected issues in the design and analysis of sport performance research. J Sports Sci 2001; 19: 811-827

4 Ballinger GA. Using generalized estimating equations for longitudinal data analysis. Organ Res Methods 2004; 7: 127-150

5 Batterham AM, Hopkins WG. Making meaningful inferences about magnitudes. Int J Sports Physiol Perform 2006; 1: 50-57
6 Benloucif S, Burgess HJ, Klerman EB, Lewy AJ, Middleton B, Murphy PJ, Parry BL, Revell VL. Measuring melatonin in humans. J Clin Sleep Med 2008; 4: 66-69

7 Booth J, Marino F, Ward JJ. Improved running performance in hot humid conditions following whole body precooling. Med Sci Sports Exerc 1997; 29: 943-949

8 Borg GAV. Psychophysical bases of perceived exertion. Med Sci Sports Exerc 1982; 14: 377-381

9 Burgess HJ, Fogg LF. Individual differences in the amount and timing of salivary melatonin secretion. PLoS One 2008; 3: e3055

10 Cagnacci A, Elliott JA, Yen SS. Melatonin: a major regulator of the circadian rhythm of core temperature in humans. J Clin Endocrinol Metab 1992; 75: 447-452

11 Cajochen $C$, Jud C, Münch M, Kobialka S, Wirz-Justice A, Albrecht U. Evening exposure to blue light stimulates the expression of the clock gene PER2 in humans. Eur J Neurosci 2006; 23: 1082-1086

12 Cajochen C, Münch M, Kobialka S, Krauchi K, Steiner R, Oelhafen $P$, Orgul S, Wirz-Justice A. High sensitivity of human melatonin, alertness, thermoregulation, and heart rate to short wavelength light. J Clin Endocrinol Metab 2005; 90: 1311-1316

13 Cotter JD, Sleivert GG, Roberts WS, Febbraio MA. Effect of pre-cooling, with and without thigh cooling, on strain and endurance exercise performance in the heat. Comp Biochem Physiol 2001; 128: 667-677

14 Deacon S, Arendt J. Posture influences melatonin concentrations in plasma and saliva in humans. Neurosci Lett 1994; 167: 191-194

15 Duffy JF, Czeisler CA. Effect of light on human circadian physiology. Sleep Med Clin 2009; 4: 165-177

16 Gonzalez-Alonso J, Teller C, Andersen SL, Jensen FB, Hyldig T, Nielsen B. Influence of body temperature on the development of fatigue during prolonged exercise in the heat. J Appl Physiol 1999; 86: 1032-1039

17 Hagan RD, Diaz FJ, Horvath SM. Plasma volume changes with movement to supine and standing positions. J Appl Physiol 1978; 45: 414-417

18 Hargreaves $M$. Physiological limits to exercise performance in the heat J Sci Med Sport 2008; 11: 66-71

19 Harriss DJ, Atkinson G. Ethical standards in sport and exercise science research: 2014 update. Int J Sports Med 2013; 34: 1025-1028

20 Hobson RM, Clapp EL, Watson P, Maughan RJ. Exercise capacity in the heat is greater in the morning than in the evening in man. Med Sci Sports Exerc 2009; 41: 174-180

21 Jay 0 , Kenny GP. Current evidence does not support an anticipatory regulation of exercise intensity mediated by rate of body heat storage. J Appl Physiol 2009; 107: 630-631

22 Krauchi K, Cajochen C, Danilenko KV, Wirz-Justice A. The hypothermic effect of late evening melatonin does not block the phase delay induced by concurrent bright light in human subjects. Neurosci Lett 1997; 232: 57-61

23 Kubota T, Uchiyama M, Suzuki H, Shibui K, Kim K, Tan X, Tagaya H, Okawa $M$, Inoue $S$. Effects of nocturnal bright light on saliva melatonin, core body temperature and sleep propensity rhythms in human subjects. Neurosci Res 2002; 42: 115-122

24 Lee DT, Haymes EM. Exercise duration and thermoregulatory responses after whole body precooling. J Appl Physiol 1995; 79: 1971-1976

25 Minors DS, Waterhouse JM, Wirz-Justice A. A human phase-response curve to light. Neurosci Lett 1991; 133: 36-40

26 Nathan PJ, Jeyaseelan AS, Burrows GO, Norman TR. Modulation of plasma melatonin concentrations by changes in posture. J Pineal Res 1998; 24: 219-223

27 Nybo L. Brain temperature and exercise performance. Exp Physiol 2012; 97: 333-339

28 O'Brien PM, O'Connor PJ. Effect of bright light on cycling performance. Med Sci Sports Exerc 2000; 32: 439-447

29 Ramanathan NL. A new weighting system for mean surface temperature of the human body. J Appl Physiol 1964; 19: 531-533

30 Reilly T, Waterhouse J. Sports performance: is there evidence that the body clock plays a role? Eur J Appl Physiol 2009; 106: 321-332

31 Schlader ZJ, Stannard SR, Mundel T. Human thermoregulatory behavior during rest and exercise - a prospective review. Physiol Behav 2010; 99: 269-275

32 Tassi P, Muzet A. Sleep inertia. Sleep Med Rev 2000; 4: 341-353

33 Wegmann M, Faude O, Poppendieck W, Hecksteden A, Frohlich M, Meyer $T$. Pre-Cooling and Sports Performance: A Meta-Analytical Review. Sports Med 2012; 42: 545-564 